\title{
Emergency Management Organizational Structure of China and Its Information Transmission Ability
}

\author{
Jian Ping \\ ${ }^{1}$ Liaoning University \\ Daoyi South Street No. 58, Shenbei New Area, Shenyang City, Liaoning Province, China 110036 \\ ${ }^{2}$ Party School of The Liaoning committee of CPC \\ Wulihe Street No. 18, Shenyang City, Liaoning Province, China 110004
}

\begin{abstract}
Information is the key to the success of the emergency management. The research suggests that the ability of information transmission is related to organizational structure in emergency management process. In order to get relatively accurate emergency response, it is necessary to change the structure of emergency organizations. In this paper, in the case of The Earthquake Relief Headquarters of the State Council, we change the current structure of emergency management organizations by adding sidelinks and shortcuts. The results show that the organizational structure with sidelinks and shortcuts has the higher ability of information transmission.
\end{abstract}

Keywords-Emergency management; Organizational structure; Information

\section{INTRODUCTION}

With the development of economy and society, unstable factors of our world surge, as well as disaster accidents occur frequently. These events seriously impact a nation's development of economy and stability of society. The national emergency management confronts great challenge.

Emergency management is complex system engineering, which was divided into three stages including prevention, response and recovery 1,2 . The mission of the emergency management is to protect the life and property of victims. Since the "9-11" attacks, governments, academics and business leaders have recognized the importance of emergency management 3 , the ability of which has become an important aspect of national security.

The complexity of emergency management determines the diversity of its response requirements. Additionally, the changing response requirements increase the difficulties of emergency management. As a result, emergency management cannot follow an inflexible plan of emergency. Researches show that effective emergency management can make a reasonable dynamic response according to the event response requirements. The key point of successful emergency management depends on the dynamic consistency between government emergency behaviors and event response requirements.

This research was financially supported by the National Natural Science Foundation of China (Grant NO. 91224003) and the Social Science Planning Funds for Liaoning (Grant NO. L16CGL016)
Successful emergency management requires a lot of information. And information transmission relies on emergency management structures. In order to control the information flow, the companies and managers are usually managed in a strict hierarchical network4,5. Moreover, the structure of emergency organization is a strict hierarchical network too. This structure is benefit for higher managers to obtain information and increase their decision-making executive ability6. However, the failure rate of nodes or links in most hierarchical networks cannot be ignored. This paper studies information flows and emergency organizational structure of China. The motivation is to improve the ability of information transmission of emergency organizations. by adding sidelinks and shortcuts.

The remain of the present paper is organized as follows. In Section 2, the ability of emergency organization obtained information is studied. In section 3 , The ability of the information transmission of the original organization and the organization with shortcuts is compared. The ability of the information transmission of the original organization and the organization with sidelinks is compared in section 4. Finally, we make a brief summary and conclusion.

\section{CURRENT STRUCTURE OF EMERGENCY MANAGEMENT ORGANIZATIONS}

Researches show that effective emergency management can make a reasonable dynamic response according to disaster information. Information transmission relies on the emergency organizational structure. Current structure of emergency management organizations of China is strict hierarchical.

\section{A. Original Model}

The paper selects The Earthquake Relief Headquarters of the State Council of China as research object, studies its organizational structure and establishes original model. As one of emergency management organizations, The Earthquake Relief Headquarters of the State Council of China is comprised of 47 members, divided into 3 levels.

A level (leader level), which is consisted of director and assistant director, is responsible for the decision-making work of earthquake relief. 
B level (coordinator level), which is consisted of 9 groups, coordinates departments to carry out the relief work.

$\mathrm{C}$ level (operator level), which is in charge of emergency management work of public emergencies.

In order to simplify our research, we assume that the emergency organizational structure can be abstracted as a network model, and call it original model "Fig. 1". Each node represents a member.

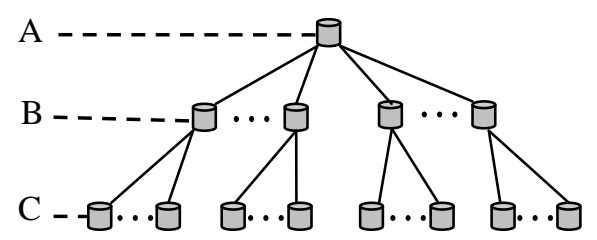

Fig. 1. Original Model.

\section{B. The Ability of Information Transmission}

Information is the necessary resource to achieve organizational objectives and provides necessary data for managers to make effective decisions. Information content is a measurement criteria of information transmission ability. Information needs to be handled when receiving, and then passes to the higher level. The processing ability of each level determines the maximum of upload information. Assuming each level has the same handling ability with a maximum of handling information $T_{B}, C$ is the information content produced by emergencies, $p$ is information transmission failure rate from one level to another; $q$ is the effective information handling rate, $q$ shows the extent of information handling. The received information content of top level, intermediate level and bottom level $C_{T}, C_{I}, C_{B}$ are listed below:

$$
\begin{gathered}
C_{T}=T_{B}\left(1-e^{-C / T_{B}}\right)(1-p)^{L-1} q^{L-1} \\
\text { Intermediate level: } \\
C_{I}=T_{B}\left(1-e^{-C / T_{B}}\right)(1-p)^{l-1} q^{l-1}
\end{gathered}
$$

Bottom level:

$$
C_{B}=C
$$

Bottom received information content $C_{B}$ is equal to produced emergency information content $C$, the information content at the top decreases along with the increasing number of network hierarchical levels, this kind of phenomenon is relevant to the information loss and processed in transmission. According to equation (1), the relationship between obtained information content at the top $C_{T}$ and bottom received information $C$ is as Figure 2 shown below.

Figure 2 chose three different information processing capacity $T_{B 1}, T_{B 2}$ and $T_{B 3}, T_{B 1}<T_{B 2}<T_{B 3}$. As seen in Figure 2, the top receiving information amount $C_{T}$ do not increase in direct ratio along with the bottom obtained information content $C$. When $C$ surpasses a certain value, $C_{T}$ will no longer increase (namely reaches maximum). $C_{T}$ maximum is related with $T_{B}$ and $L$, the smaller $T_{B}$ and the greater $L$ is, $C_{T}$ becomes greater.

The results show that, in the current emergency organization, we can improve $C_{T}$ by changing $L . C_{T}$ is a measurement criteria of information transmission ability. $L$ is for the organizational structure. This means the ability of information transmission is related to organizational structure. In the situation, in order to get relatively accurate emergency response, that is necessary to improve the ability of information transmission by changing organizational structure "Fig. 2".

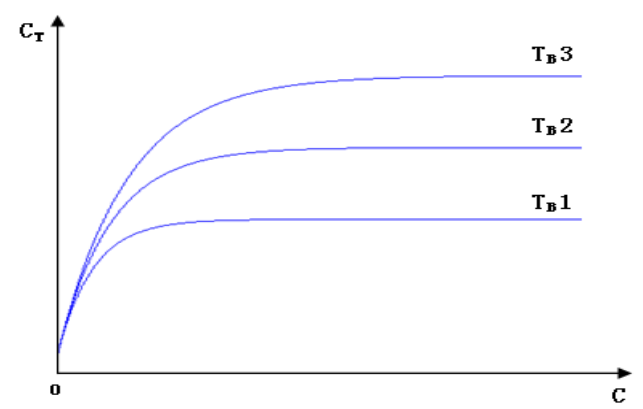

Fig. 2. The relationship between obtained information content at the top and receiving information at the bottom.

\section{THE EMERGENCY ORGANIZATIONAL STRUCTURE WITH SHORTCUTS}

In emergency management, the ability of information transmission is related to the emergency organizational structure. In this section, that will be discussed to improve the ability of information transmission through adding shortcuts in the current emergency organizational structure.

\section{A. The Organizational Structure Model with Shortcuts (Shortcuts Model)}

The current emergency organizational structure is strict hierarchical, which benefits the execution of high-level decision-making. However, it often shows some disadvantages:

- Information loss in transmission, emergencies cause a failure of connection in an organization department or between different departments.

- Information distortion in transmission, information is transmitted from the sender to the receiver by levels.

- These disadvantages reduce the ability of information transmission. 


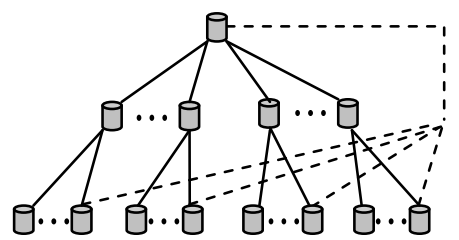

Fig. 3. Network Model of Emergency Management Structure with shortcuts.

In order to improve the ability of information transmission, we develop a new organization structure model with shortcuts "Fig. 3". When some information link does not function properly, or the capacity of some information channel is not high enough, an alternative information link should be established. Based on the existing structure, the model in Figure 3 creates shortcuts for the departments, which are affected by emergencies, from the bottom to the top. In some sense, it is recommended to establish a new link (a "shortcut"), if it would reduce information flows via many nodes, i.e., if it would reduce detours ${ }^{7}$.

\section{B. Information Transmission Accessibility}

Information transmission accessibility is the probability of information transmission from a department in the bottom to the top. In emergency management, emergency events often destroy the communication channels in a department or between different organization departments, and then cause disruptions of information transmission. Within an organization, higher information transmission accessibility is, more hardly its transmission will be interrupted. Information transmission accessibility $M$ can be expressed as:

$$
\begin{cases}M=1-\prod_{X=1}^{S}\left(1-(1-p)^{L_{X}-1}\right) & (s=1,2,3, \ldots, k) \\ M=0 & \left(s=0, L_{X} \rightarrow \infty\right)\end{cases}
$$

In equation (2), $s$ is the number of the channels of information transmission from a department in the bottom to the top. Information transmission accessibility can be divided into two kinds of situations:

- Emergency destroyed the communication channels in a department or between different departments, causing no information channel $(s=0)$. On this occasion, the hierarchical levels from bottom to top could be treated as infinity $\left(L_{x} \rightarrow \infty\right)$, and the information transmission accessibility $M=0$.

- There are communication channels between bottom and top in organization $(s=1,2,3, \ldots, k)$. When information transmission failure rate $p$ is fixed, the communication channels $(s)$ are more, and the information transmission accessibility is larger; $L_{x}$ is the bigger, the information transmission accessibility is smaller.
- In two situations, assuming the number of hierarchical levels $L=3$, transmission loss rate $p=0.2$, according to equation (2), the accessibility is as shown in "Table I".

TABLE I. INFORMATION TRANSMISSION ACCESSIBILITY OF Original Model ANd SHORTCUtS Model (When $L=3, p=0.2$ )

\begin{tabular}{c|l|l}
\hline & \multicolumn{1}{|c|}{$\begin{array}{c}\text { Completely } \\
\text { destroyed }\end{array}$} & Not completely destroyed \\
\hline Original model & 0 & 0.64 \\
Shortcuts model & 0.8 & 0.936 \\
\hline
\end{tabular}

In two situations, information transmission ability completely destroyed or not completely destroyed, the information transmission accessibility in the shortcuts model is higher than the one in the original model.

\section{The Quantity of Fidelity Information Received by the \\ Top Level}

Information can be distorted within an organization. Improving the ability of information transmission requires decreasing the information transmission distortion. Fidelity of information transmission $C_{R}$ indicates information received by the top level, it can be expressed as:

$$
C_{R}=C_{T}(1-R)^{L-1}
$$

In the equation, $R$ is the information distortion rate; $C_{T}$ is the quantity of information received by the top; $L$ is the number of hierarchical levels. $C_{R}$ can measure the reliability of information transmission.

In shortcuts model, partial information can be transmitted by shortcuts. Assuming $f$ is the ratio of the information quantity transmitted by the shortcut from the bottom to the top and the total information quantity received by the top. In shortcuts model, $C_{R}{ }^{\prime}$ is the quantity of the fidelity information received by the top level. There are two parts in equation (4), one is the quantity of fidelity information in original channels, the other is the quantity of fidelity information in the shortcut. $C_{R}{ }^{\prime}$ could be calculated from both parts added together.

$$
C_{R}^{\prime}=C_{T}(1-f)(1-R)^{L-1}+C_{T} f(1-R)
$$

Assuming information transmission distortion rate $R=0.1, f=0.3$. According to equation (3) and (4), the quantities of the fidelity information received by the top level are as shown in "Table II".

TABLE II. THE QUANTITIES OF THE FIDELITY INFORMATION RECEIVED BY THE TOP LEVEL

\begin{tabular}{c|c}
\hline & Fidelity information quantities \\
\hline Original model & $0.81 C_{T}$ \\
Improved model & $0.837 C_{T}$ \\
\hline
\end{tabular}

By Table 2, the quantity in the shortcuts model is higher than the one in the original model. Through comparisons above, the accessibility in information transmission and the 
quantities of the fidelity information received by the top level in shortcuts model are all higher than them in the original model. It proves that the organization with shortcuts has a higher information transmission ability.

\section{THE EMERGENCY ORGANIZATIONAL STRUCTURE WITH SIDELINKS}

The hierarchical organization form has departments of subordinates, which would not have information flows among each other nor to the outside. This organization is vulnerable to the failure of links or nodes. If, for example, one of the subordinates is ill or on holidays, or moves to another company, nobody can easily take over his or her tasks and responsibilities ${ }^{7}$.

\section{The Organizational StRUCtURE MODEL With SIDELINKS (SIDELINKS MODEL)}

In organizations, quality of information transmission is better, the ability of information transmission is higher. In order to improve the ability of information transmission, in the section, we will study the relationship between the quality of information transmission and organizational structure.

The Earthquake Relief Headquarters of the State Council of China consists of nine groups. We assume that all members in each group link up with each other. According to the original model, To establish organizational structure model with sidelinks "Fig. 4". Each node represents a member.

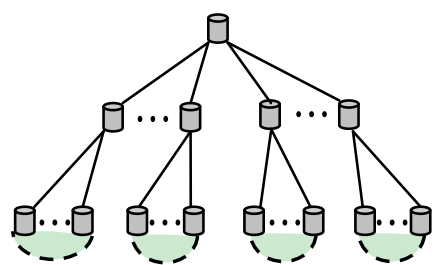

Fig. 4. Network Model of Emergency Management Structure with sidelinks.

\section{A. The Quality of Information}

The quality is used to measure the accuracy degree of the information flowing in the system or between elements. The quality entropy can measure the uncertainty of the information quality. The higher quality of information transmission in organization, the better structure the organization has.

The quality of information can be calculated as the following formula:

$$
R_{2}=1-H_{2} / H_{2 m} R_{2} \in[0,1]^{8}
$$

In formula $5, R_{2}$ is the quality of information, $H_{2 m}$ is the maximum of the quality entropy, $\mathrm{H}_{2}$ is the total of the quality entropy.

$$
H_{2 m}=\log _{2} A_{2}
$$

$A_{2}$ is the total number of the system micro-state (the system micro-state is the number state of some members when observed the system from one aspect).

$$
A_{2}=\sum_{i=1}^{N} K_{i}
$$

$K_{i}$ represents degree of node $\mathrm{i}$, is the number of nodes contacting with the node $\mathrm{i}$ directly ( $\mathrm{i}$ is the node number, $\mathrm{i}=1,2, \ldots \ldots, \mathrm{N})$.

$$
H_{2}=\sum_{i=1}^{N} H_{2}(i)
$$

$H_{2}(i)$ is the quality entropy of the node $i$, describes the uncertainty of opportunities for errors in the information transmission process of this node.

$$
H_{2}(i)=-P_{2}(i) \log _{2} P_{2}(i) \quad i=1,2, \ldots, N
$$

$P_{2}(i)$ is the probability for the node $\mathrm{i}$ to achieve the

$$
\text { quality micro-state. }
$$

$$
P_{2}(i)=K_{i} / A_{2}
$$

According to the above formula, the quality of information transmission in the two models is obtained, as shown in "Table III"

TABLE III. THE QUALITY OF INFORMATION TRANSMISSION

\begin{tabular}{ccccc}
\hline $\begin{array}{c}\text { network } \\
\text { model }\end{array}$ & $A_{2}$ & $H_{2}$ & $H_{2 m}$ & $R_{2}$ \\
\hline $\begin{array}{c}\text { original } \\
\text { model }\end{array}$ & 140 & 5.016 & 7.129 & 0.296 \\
$\begin{array}{c}\text { Sidelinks } \\
\text { model }\end{array}$ & 702 & 5.391 & 9.455 & 0.430 \\
\hline
\end{tabular}

According to "Table III", the value of the quantity in the sidelinks model is higher than the one in the original model. This suggests that the increase of sidelinks between the organization members can improve the ability of information transmission.

\section{CONCLUSION}

Considering the limited information processing capacity of human beings, hierarchical forms of organization seems to be the most efficient, if all its elements work perfectly and there is enough time for information transmission. The current organizational structure of emergency management of China is hierarchical. However, hierarchical organizations are vulnerable to the failure of links or nodes. In order to solve the above problems, In the case of The Earthquake Relief Headquarters of the State Council, We have established original model of the current structure of emergency organizations and modified the model by adding sidelinks and shortcuts. Using accessibility, fidelity and quality of information transmission to measure the performance, we conclude that sidelinks and shortcuts can significantly improve the ability of information transmission of emergency organizations. 


\section{REFERENCE}

[1] William L. Waugh, "Living with Hazards, Dealing with Disasters: An Introduction to Emergency Management”, M. E. Sharpe, USA, 2000.

[2] Dag K.J.E. Von Lubitz, James E. Beakley, Frederic Patricelli, “All Hazards Approach to Disaster Management: the Role of Information and Knowledge Management, Boyd's OODA Loop, and Networkcentricity", Disasters, vol. 32, no.4, pp.561-585, 2008.

[3] Yossi Sheffi, "Supply Chain Management under the Threat of International Terrorism", International Journal of Logistics Management, vol. 12, no. 2, pp.1-11, 2001.

[4] Erzsebet Ravasz, Albert-Laszlo Barabasi, "Hierarchical Organization in Complex Networks", Physical Review, vol. 67, no.2, pp. 1-7, 2003.

[5] Raj Kumar Pan, Sitabhra Sinha, "Modular Networks with Hierarchical Organization: the Dynamical Implications of Complex Structure", Journal of Physics, vol.71, no. 2, pp. 331-340, 2008.

[6] Juan A. Almendral, Luis Lopez, Miguel A.F. Sanjuan, "Information Flow in Generalized Hierarchical Networks", Physica A, vol.324, no. 1, pp.424-429, 2003.

[7] Dirk Helbing, Hendrik Ammoser, Christian Kuhnert, "Information Flows in Hierarchical Networks and the Capability of Organizations to Successfully Respond to Failures, Crises, and Disasters", Physica A, vol. 363, no.1, 141-15, 2006

[8] Yan Zhilin, Qiu Wanhua, Chen Zhiqiang. Evaluation of System Order Degree as Viewed from Entropy, Systems Engineering- theory \& Practice, (6) (1997)45-48. 